\title{
ElectronTransfer Induced Ring Opening of $\alpha$-Epoxyketones: Spirodioxolane Formation
}

\author{
Hamid R. Memarian* and Farzad Nikpour \\ University of Isfahan, Faculty of Sciences, Department of Chemistry, 81744 Isfahan, Iran. Tel: (+98) \\ 311-793 2707, Fax: (+98) 311-668 9732.
}

* Author to whom correspondence should be addressed, e-mail: memarian@sci.ui.ac.ir

Received: 21 July 2001; in revised form: 17 November 2001 / Accepted: 13 December 2001 / Published: 31 January 2002

\begin{abstract}
Stereospecific formation of spirodioxolanes has been observed on electron transfer induced ring opening of $\alpha$-epoxyketones by 2,4,6-triphenylpyrylium tetrafluoroborate in the presence of cyclohexanone.
\end{abstract}

Keywords: Dioxolanes; Electron transfer; $\alpha$-Epoxyketones; Photochemistry; Spiro compounds.

\section{Introduction}

Ring opening of epoxides and $\alpha$-epoxyketones induced by electron transfer to suitable electron acceptors in the presence of various nucleophiles leads to the formation of some interesting compounds such as ethers, dioxolanes and trioxolanes. Such reactions have been recognized as important processes not only in thermal but also in photochemical reactions.

Single electron transfer (SET) induced ring opening reactions of epoxides [1-4], $\alpha$-epoxyketones [5-8] and $\alpha$-epoxyesters [9] have demonstrated $\mathrm{C}_{\alpha}-\mathrm{C}_{\beta}, \mathrm{C}_{\alpha}-\mathrm{O}$ and $\mathrm{C}_{\beta}-\mathrm{O}$ bond cleavage through photosensitized electron transfer by dicyanoanthracene [1,2], tetracyanoethylene [3,4] and 2,4,6triphenylpyrylium tetrafluoroborate [5-8] or thermally induced electron transfer to ceric ammonium nitrate [10,11], 2,3-dichloro-5,6-dicyano-p-benzoquinone [12] and iron (III) chloride [13]. 
Previously we investigated the formation of charge-transfer complexes between some $\alpha$-epoxyketones as donor molecules and tetracyanoethylene (TCNE) as the acceptor molecule [14]. These results indicated that the amount of CT-complex formation is dependent on the extent of electron donating character of the $\alpha$-epoxyketones, which is directly related to the nature and location of the additional substituent on the parent molecule. Recently we reported on the electron transfer induced ring opening of some $\alpha$-epoxyketones of interest (1a-f) using 2,4,6-triphenylpyrylium tetrafluoroborate (2) as photocatalyst in methanol [8] and acetone solutions [15] (Scheme 1). The aim of this work was to explain the effects of the type of the additional substituent on the parent molecule (1a) and the nature of the nucleophile used as solvent on the rate of the ring opening process.

\section{Scheme 1}

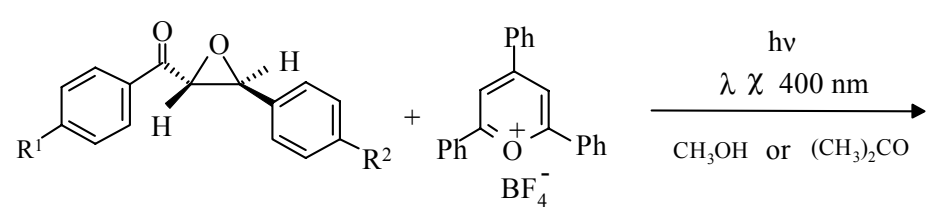

1

2

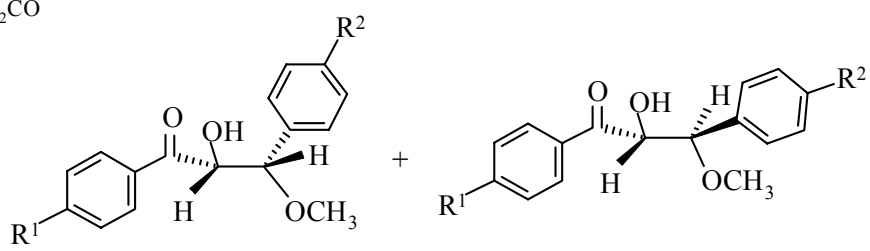

\begin{tabular}{c|c|c}
$\mathbf{1}$ & $\mathrm{R}^{1}$ & $\mathrm{R}^{2}$ \\
\hline $\mathbf{a}$ & $\mathrm{H}$ & $\mathrm{H}$ \\
$\mathbf{b}$ & $\mathrm{H}$ & $\mathrm{CH}_{3}$ \\
$\mathbf{c}$ & $\mathrm{CH}_{3}$ & $\mathrm{H}$ \\
$\mathbf{d}$ & $\mathrm{H}$ & $\mathrm{OCH}_{3}$ \\
$\mathbf{e}$ & $\mathrm{OCH}_{3}$ & $\mathrm{H}$ \\
$\mathbf{f}$ & $\mathrm{OCH}_{3}$ & $\mathrm{OCH}_{3}$ \\
g & $\mathrm{Br}$ & $\mathrm{H}$ \\
$\mathbf{h}$ & $\mathrm{H}$ & $\mathrm{Br}$
\end{tabular}
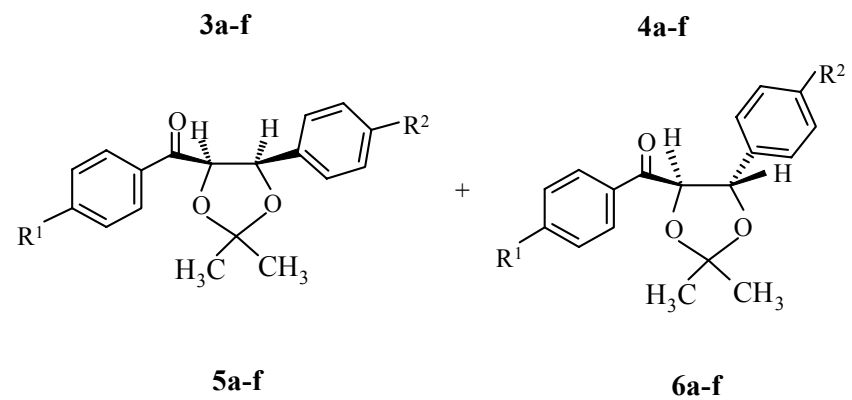

Although the rate of the ring opening in methanol was faster than in acetone, a diastereoselective reaction has been observed in the latter solutions. As a continuation of this work we have now investigated this reaction in the presence of cyclohexanone as nucleophile. By also using a cyclic ketone in place of acetone, we have attempted to elucidate the effect of the steric hindrance of the nucleophile on the diastereoselectivity of reaction and also the effect of the extent of its nucleophilicity on the rate of the ring opening.

\section{Results and discussion}

Irradiation $(\lambda \geq 400 \mathrm{~nm})$ of each $\alpha$-epoxyketones (1a-e, $\mathbf{1 g})$ and (2) in molar ratio of 10:1 in cyclohexanone / acetonitrile $(19 \mathrm{ml} / 1 \mathrm{ml})$ resulted in the opening of the epoxide ring and formation of a sole 
product of type 7 in each reaction (Scheme 2). It should be mentioned that the light has been passed through a filter solution to prevent the absorption of light by compounds 1a-e and 1g, therefore selective excitation of the photocatalyst $\mathbf{2}$ has been achieved.

\section{Scheme 2}<smiles>[R]c1ccc(C(=O)[C@@H]2O[C@H]2c2ccc([R4])cc2)cc1</smiles>

1

2 7

\begin{tabular}{c|c|c}
$\mathbf{1}$ & $\mathrm{R}^{1}$ & $\mathrm{R}^{2}$ \\
\hline $\mathbf{a}$ & $\mathrm{H}$ & $\mathrm{H}$ \\
$\mathbf{b}$ & $\mathrm{H}$ & $\mathrm{CH}_{3}$ \\
$\mathbf{c}$ & $\mathrm{CH}_{3}$ & $\mathrm{H}$ \\
$\mathbf{d}$ & $\mathrm{H}$ & $\mathrm{OCH}_{3}$ \\
$\mathbf{e}$ & $\mathrm{OCH}_{3}$ & $\mathrm{H}$ \\
$\mathbf{g}$ & $\mathrm{Br}$ & $\mathrm{H}$ \\
$\mathbf{h}$ & $\mathrm{H}$ & $\mathrm{Br}$
\end{tabular}

IR and ${ }^{1} \mathrm{H}-\mathrm{NMR}$ data provided useful information for the structural assignment of the products (7a-e, $7 \mathbf{g}$ ). Monitoring of the photoreaction/conversion of $1 \mathbf{a}-\mathbf{e}, \mathbf{1 g}$ and formation of $7 \mathbf{a}-\mathbf{e}, \mathbf{7 g}$ was carried out using IR by following the appearance of the $\mathrm{CH}$-stretching of the cyclohexane ring at 2800-2980 and a shift of the CO bond to higher frequency due to opening of the epoxide ring and the resulting release of the ring strain. The results are summarized in Table 1.

Table1. Comparison of the CO-stretching in the IR spectra of compounds 1a-e, $1 \mathrm{~g}$ with those of $7 \mathbf{a}-\mathbf{e}, 7 \mathrm{~g}$

\begin{tabular}{|c|c|c|c|}
\hline Compd. & $\mathrm{CO}\left(\mathrm{cm}^{-1}\right)$ & Prod. & $\mathrm{CO}\left(\mathrm{cm}^{-1}\right)$ \\
\hline 1a & 1670 & $\mathbf{7 a}$ & 1695 \\
1b & 1660 & $\mathbf{7 b}$ & 1680 \\
1c & 1665 & $\mathbf{7 c}$ & 1685 \\
1d & 1660 & $\mathbf{7 d}$ & 1690 \\
1e & 1665 & $\mathbf{7 e}$ & 1680 \\
$\mathbf{1 g}$ & 1670 & $\mathbf{7 g}$ & 1690 \\
\hline
\end{tabular}

The ${ }^{1} \mathrm{H}-\mathrm{NMR}$ spectra showed a multiplet at $\sim 1.1-2.4 \mathrm{ppm}$ with an integration corresponding to $10 \mathrm{H}$ for the cyclohexyl moiety and an $\mathrm{AB}$-system for the $3-\mathrm{H}$ and $4-\mathrm{H}$ of the dioxolane ring. A cis 
orientation of the hydrogens of the dioxolane ring was confirmed by comparison of their chemical shifts and coupling constants with those of known dioxolanes [16]. Semiempirical PM3 calculations on dioxolanes $\mathbf{7 a - e , ~} \mathbf{7 g}$, showed that, for example for 7a, in the optimized structure (Figure 1) 4-H lies in the same plane as the phenyl ring. Since the anisotropic effect of the phenyl ring causes a deshielding on this proton, therefore the resonance of 4-H should appear at a lower field in comparison to the resonance for the benzylic protons $[17,18]$. On the other hand, the inductive effect of the benzoyl group on 3-H leads to absorption of this proton at lower field, therefore, the chemical shifts of 3-H and 4-H must be closer to each other. (Table 2)

Table 2. Structurally relevant ${ }^{1} \mathrm{H}-\mathrm{NMR}$ chemical shifts ( $\delta$ values) and $J^{1} \mathrm{H}-{ }^{1} \mathrm{H}$ coupling constants $[\mathrm{Hz}]$ of $(\mathbf{7 a - e ,}, \mathbf{7 g})$

\begin{tabular}{|l|c|c|c|}
\hline Compd. & $\delta_{\mathrm{A}}$ & $\delta_{\mathrm{B}}$ & $J_{\mathrm{AB}}$ \\
\hline $\mathbf{7 a}$ & $4-\mathrm{H}$ & $3-\mathrm{H}$ & \\
$\mathbf{7 b}$ & 5.52 & 5.80 & 8.0 \\
$\mathbf{7 c}$ & 5.49 & 5.78 & 7.6 \\
$\mathbf{7 d}$ & 5.59 & 5.74 & 7.6 \\
$\mathbf{7 e}$ & 5.50 & 5.77 & 7.6 \\
$\mathbf{7 g}$ & 5.60 & 5.74 & 7.4 \\
\hline
\end{tabular}

Figure 1. Optimized structure of 7a using PM3 calculations

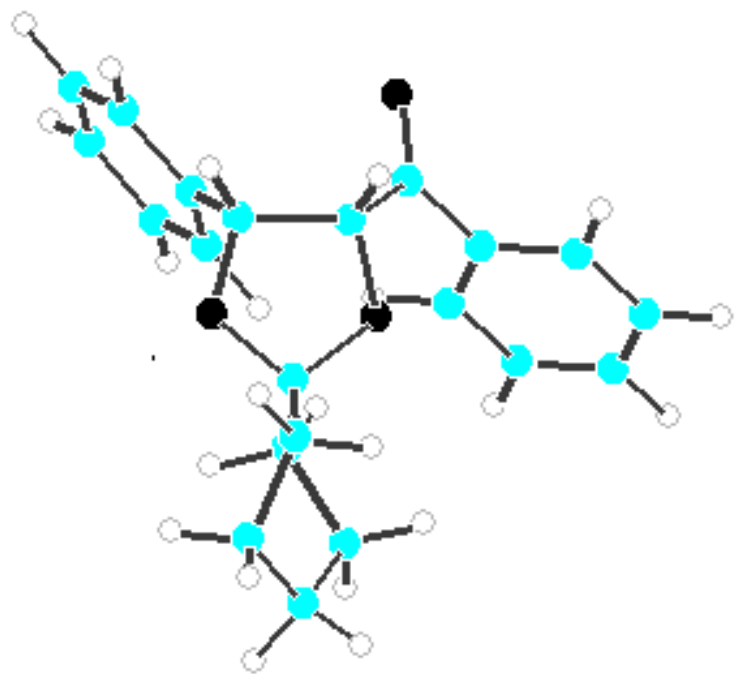

The mechanism of the ring opening can be explained as follows: due to electron transfer from 1a-e and $\mathbf{1 g}$ to photoexcited $\mathbf{2}$, the radical cations $\mathbf{8}$ and $\mathbf{9}$ could be formed, which are then trapped by 
nucleophilic attack of cyclohexanone. The observed high diastereospecificity of reaction supports the involvement of the intermediate 8 rather than 9, which should be formed better in apolar cyclohexanone solution and would be attacked preferably by cyclohexanone acting as a nucleophilic reagent at the carbon atom at the rear of the bond comprising the leaving group oxygen due to the steric hindrance of the nucleophile. This suggestion is also supported by the formation of single products, namely the cis-products $7 \mathbf{a}-\mathbf{e}, \mathbf{7 g}$.

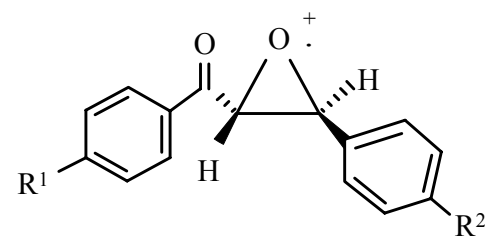

8

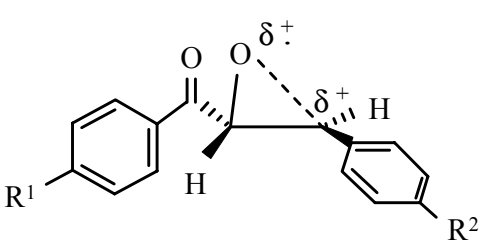

9

The electron transfer induced ring opening and the involvement of the intermediates such as 8 or $\mathbf{9}$ are supported by the following basis:

1. Irradiation of $\mathbf{1 a - e}$ and $\mathbf{1 g}$ in the absence of the photocatalyst $\mathbf{2}$ at $\lambda \geq 400 \mathrm{~nm}$ (blank experiment) does not result the formation of any product.

2. Upon irradiation of $\mathbf{1 h}$ and $\mathbf{2}$ in the presence of cyclohexanone unchanged starting materials were obtained even after 15 hours irradiation. This indicates that the formation of $\mathbf{8}$ or $\mathbf{9}$ will be destabilized by the electron withdrawing character of the bromine on the phenyl ring directly attached to the epoxide ring. As we show in Table 3, the ring opening will be facilitated, when the phenyl group bears the electron donor group such as methyl or methoxy group (1b, 1d).

Table 3. Photochemical reactions of 1a-e, $1 \mathrm{~g}$ catalyzed by

(2) in cyclohexanone solution ${ }^{a}$

\begin{tabular}{|c|c|c|c|}
\hline Compd. & Irrad. Time $^{\mathrm{b}}(\mathrm{hr})$ & Product & Yield \% $^{\mathrm{c}}$ \\
\hline 1a & 5 & $\mathbf{7 a}$ & 27 \\
$\mathbf{1 b}$ & 2.5 & $\mathbf{7 b}$ & 31 \\
$\mathbf{1 c}$ & 5 & $\mathbf{7 c}$ & 26 \\
$\mathbf{1 d}$ & 2 & $\mathbf{7 d}$ & 33 \\
$\mathbf{1 e}$ & 5 & $\mathbf{7 e}$ & 30 \\
$\mathbf{1 g}$ & 15 & $\mathbf{7 g}$ & 24 \\
\hline
\end{tabular}

${ }^{\mathrm{a}}$ Molar ratio of (1a-e, 1g) : (2) (10:1), [1a-e, 1g] $=0.04 \mathrm{M}$ and $[2]=0.004 \mathrm{M} .{ }^{b}$ Times are given after maximum progression of the reaction. ${ }^{\mathrm{c}}$ Isolated yields, based on consumed $\mathbf{1}$. 
It should be mentioned that some unidentified products were formed, which could not be isolated in pure form for spectroscopic analysis. The formation of the trans isomer was ruled out based on comparison of the TLC of the cis isomer with the TLC of the reaction mixture. The formation of the cis-products obtained in cyclohexanone solution compared to the results obtained in acetone can be attributed due to the steric hindrance of nucleophile.

\section{Acknowledgments}

This research was supported by research grants from the Isfahan University Research Council (Grant no. 77312).

\section{Experimental}

\section{General}

Melting points were determined on a Stuart Scientific SMP-2 capillary apparatus and are uncorrected. IR spectra were recorded using $\mathrm{KBr}$ pellets on a Shimadzu IR-435 spectrometer. ${ }^{1} \mathrm{H}-\mathrm{NMR}$ spectra were recorded on a Bruker AW 80 spectrometer in $\mathrm{CDCl}_{3}$ with TMS as internal standard. Mass spectra were measured by electron impact at $70 \mathrm{eV}$ on a Micromass Platform instrument.. Elemental analyses were measured on a CHN-O-RAPID instrument. Preparative thin layer chromatography (PTLC) was carried out on $20 \times 20 \mathrm{~cm}^{2}$ plates, coated with a $1 \mathrm{~mm}$ layer of Merck silica gel $\mathrm{PF}_{254}$, prepared by applying the silica as a slurry and drying in air. All irradiations were carried out using a $400 \mathrm{~W}$ high-pressure Hg-vapor lamp from NARVA in Duran glass equipment with sample cooling by cold running water. The light has been passed through a filter solution $\left(75 \mathrm{gL}^{-1}\right.$ of $\mathrm{NaNO}_{2}$ and $4.4 \mathrm{gL}^{-1}$ of $\mathrm{CuSO}_{4}$ in $2.7 \mathrm{M} \mathrm{NH}_{4} \mathrm{OH}$ ) [19] to obtain $\lambda \geq 400 \mathrm{~nm}$ for the selective excitation of the photocatalyst $\mathbf{2}$ and also to prevent the absorption of the light by $\mathbf{1 a - e , ~} \mathbf{1 g - h}$.

General experimental procedure for the conversions of $\mathbf{1 a - e , ~} \mathbf{1 g - h}$ by photoexcited $\mathbf{2}$ in the presence of cyclohexanone.

To a solution of $0.08 \mathrm{mmol}$ of $\mathbf{2}$ in dry acetonitrile $(1 \mathrm{~mL})$ and freshly distilled cyclohexanone (19 $\mathrm{mL}, \mathrm{c}=0.004 \mathrm{M})$ was added $0.8 \mathrm{mmol}$ of each of compounds 1a-e, 1g-h $(\mathrm{c}=0.04 \mathrm{M})$ and the mixtures were irradiated until maximum progression of the reaction was observed. The excess of cyclohexanone was evaporated under reduced pressure and the residue was purified by preparative TLC (PTLC).

Reaction of 1,3-diphenyl-2,3-epoxy-1-propanone (1a). PTLC (12: 1 petroleum ether-ethyl acetate, three times): Zone 1, recovered 1a (35 mg, 20\%); Zone 2, $69 \mathrm{mg}$ (27\%) of cis-3-benzoyl-4-phenyl-2,5dioxaspiro[4,5]decane (7a), recrystallized from cyclohexane, m.p. 95-98 ${ }^{\circ} \mathrm{C}$; IR $\mathrm{cm}^{-1}: 3055,2880$ - 
2990, 1695, 1590, 1220, 1110, 1080; ${ }^{1} \mathrm{H}-\mathrm{NMR}(\delta): 1.10-2.42(\mathrm{~m}, 10 \mathrm{H}$, cyclohexane H), AB-system $\left(\delta_{\mathrm{A}}=5.52, \delta_{\mathrm{B}}=5.80,{ }^{3} \mathrm{~J}_{\mathrm{AB}}=8.0 \mathrm{~Hz}, 2 \mathrm{H}, 4-\mathrm{H}, 3-\mathrm{H}\right), 7.03(\mathrm{~s}, 5 \mathrm{H}$, phenyl ring $\mathrm{H}), 7.15-7.44 \mathrm{ppm}(\mathrm{m}, 5 \mathrm{H}$, benzoyl group); EI-MS m/z (\%): $322\left(\mathrm{M}^{+}, 1\right), 224\left(\mathrm{M}^{+}-\mathrm{C}_{6} \mathrm{H}_{10} \mathrm{O}, 15\right), 217\left(\mathrm{M}^{+}-\mathrm{C}_{6} \mathrm{H}_{5} \mathrm{CO}, 9\right), 119\left(\mathrm{M}^{+}-\right.$ $\left.\mathrm{C}_{6} \mathrm{H}_{10} \mathrm{O},-\mathrm{C}_{6} \mathrm{H}_{5} \mathrm{CO}, 30\right), 105\left(\mathrm{C}_{6} \mathrm{H}_{5} \mathrm{CO}^{+}, 100\right), 77\left(\mathrm{C}_{6} \mathrm{H}_{5}{ }^{+}, 28\right)$; Anal. Calcd. for $\mathrm{C}_{21} \mathrm{H}_{22} \mathrm{O}_{3}(322.40)$ : C, 78.23; H, 6.88. Found: C, 77.98; H, 6.93.

Reaction of 3-p-methylphenyl-1-phenyl-2,3-epoxy-1-propanone (1b). PTLC (12:1 petroleum etherethyl acetate, four times): Zone 1, recovered $1 \mathbf{b}$ (19 mg, 10\%); Zone 2, $84 \mathrm{mg}$ (31\%) of cis-3-benzoyl4-(p-methylphenyl)-2,5-dioxaspiro[4,5]decane (7b), recrystallized from cyclohexane, m.p. 114-116 ${ }^{\circ} \mathrm{C}$; IR cm ${ }^{-1}: 3045,2880-2990,1680,1590,1200,1150,1080 ;{ }^{1} \mathrm{H}-\mathrm{NMR}(\delta): 1.15-2.20(\mathrm{~m}, 10 \mathrm{H}$, cyclohexane $\mathrm{H}), 2.14\left(\mathrm{~s}, 3 \mathrm{H}, \mathrm{CH}_{3}\right), \mathrm{AB}$-system $\left(\delta_{\mathrm{A}}=5.46, \delta_{\mathrm{B}}=5.78,{ }^{3} \mathrm{~J}_{\mathrm{AB}}=7.6 \mathrm{~Hz}, 2 \mathrm{H}, 4-\mathrm{H}, 3-\mathrm{H}\right)$, 6.72-7.58 ppm (m, 9H, aromatic H); EI-MS m/z (\%): $336\left(\mathrm{M}^{+}, 4\right), 238\left(\mathrm{M}^{+}-\mathrm{C}_{6} \mathrm{H}_{10} \mathrm{O}, 75\right), 231\left(\mathrm{M}^{+}-\right.$ $\left.\mathrm{C}_{6} \mathrm{H}_{5} \mathrm{CO}, 43\right), 216\left(\mathrm{M}^{+}-\mathrm{CH}_{3} \mathrm{C}_{6} \mathrm{H}_{4} \mathrm{CHO}, 68\right), 133\left(\mathrm{M}^{+}-\mathrm{C}_{6} \mathrm{H}_{5} \mathrm{CO},-\mathrm{C}_{6} \mathrm{H}_{10} \mathrm{O}, 84\right), 119\left(\mathrm{CH}_{3} \mathrm{C}_{6} \mathrm{H}_{4} \mathrm{CO}^{+}, 48\right)$, $105\left(\mathrm{C}_{6} \mathrm{H}_{5} \mathrm{O}^{+}, 100\right), 91\left(\mathrm{CH}_{3} \mathrm{C}_{6} \mathrm{H}_{5}^{+}, 55\right), 77\left(\mathrm{C}_{6} \mathrm{H}_{5}{ }^{+}\right.$, 77); Anal. Calcd. for $\mathrm{C}_{22} \mathrm{H}_{24} \mathrm{O}_{3}$ (336.43): C, 78.54; H, 7.19. Found: C, 77.94; H, 7.21.

Reaction of 1-p-methylphenyl-3-phenyl-2,3-epoxy-1-propanone (1c). PTLC (12:1 petroleum etherethyl acetate, three times): Zone 1, recovered 1c (38 mg, 20\%); Zone 2, $70 \mathrm{mg}$, (26\%) of cis-3-(pmethylbenzoyl)-4-phenyl-2,5-dioxaspiro[4,5]decane (7c), recrystallized from cyclohexane, m.p. 116$118{ }^{\circ} \mathrm{C}$; IR cm ${ }^{-1}: 3045,2880-2990,1685,1610,1250,1105 ;{ }^{1} \mathrm{H}-\mathrm{NMR}(\delta): 1.15-2.18$ (m, $10 \mathrm{H}$, cyclohexane $\mathrm{H}), 2.22\left(\mathrm{~s}, 3 \mathrm{H}, \mathrm{CH}_{3}\right), \mathrm{AB}$-system $\left(\delta_{\mathrm{A}}=5.49, \delta_{\mathrm{B}}=5.74,{ }^{3} \mathrm{~J}_{\mathrm{AB}}=7.6 \mathrm{~Hz}, 2 \mathrm{H}, 4-\mathrm{H}, 3-\mathrm{H}\right)$, AB-system $\left(\delta_{\mathrm{A}}=6.95, \delta_{\mathrm{B}}=7.37,{ }^{3} \mathrm{~J}_{\mathrm{AB}}=7.6 \mathrm{~Hz}, 4 \mathrm{H}\right.$, phenyl ring $\left.\mathrm{H}\right), 7.05 \mathrm{ppm}(\mathrm{s}, 5 \mathrm{H}$, aromatic H); EIMS: $m / z(\%): 336\left(\mathrm{M}^{+}, 5\right), 238\left(\mathrm{M}^{+}-\mathrm{C}_{6} \mathrm{H}_{10}, 32\right), 230\left(\mathrm{M}^{+}-\mathrm{C}_{6} \mathrm{H}_{5} \mathrm{CHO}, 19\right), 217\left(\mathrm{M}^{+}-\mathrm{CH}_{3} \mathrm{C}_{6} \mathrm{H}_{4} \mathrm{CO}, 35\right)$, $119\left(\mathrm{CH}_{3} \mathrm{C}_{6} \mathrm{H}_{4} \mathrm{CO}^{+}, 100\right), 105\left(\mathrm{C}_{6} \mathrm{H}_{4} \mathrm{CO}^{+}, 46\right), 91\left(\mathrm{CH}_{3} \mathrm{C}_{6} \mathrm{H}_{4}{ }^{+}, 74\right), 77\left(\mathrm{C}_{6} \mathrm{H}_{5}{ }^{+}, 35\right)$; Anal. Calcd. for $\mathrm{C}_{22} \mathrm{H}_{24} \mathrm{O}_{3}$ (336.43): C, 78.54; H, 7.19. Found: C, 78.01; H, 7.15.

Reaction of 3-p-methoxyphenyl-1-phenyl-2,3-epoxy-1-propanone (1d). PTLC (10:1 petroleum etherethyl acetate, three times): Zone 1, recovered 1d (20 mg, 10\%); Zone 2, $95 \mathrm{mg}$ (33\%) of cis-3-benzoyl4-(p-methoxyphenyl)-2,5-dioxaspiro[4,5]decane (7d), recrystallized from cyclohexane, m.p. 77-78 ${ }^{\circ} \mathrm{C}$; IR cm${ }^{-1}$ : 3040, 2880-2990, 1690, 1610, 1580, 1240, 1170, 1100; ${ }^{1} \mathrm{H}-\mathrm{NMR}(\delta): 1.10-2.15$ (m, 10H, cyclohexane $\mathrm{H}), 3.64\left(\mathrm{~s}, 3 \mathrm{H}, \mathrm{OCH}_{3}\right), \mathrm{AB}$-system $\left(\delta_{\mathrm{A}}=5.49, \delta_{\mathrm{B}}=5.77,{ }^{3} \mathrm{~J}_{\mathrm{AB}}=7.6 \mathrm{~Hz}, 2 \mathrm{H}, 4-\mathrm{H}, 3-\mathrm{H}\right)$, AB-system $\left(\delta_{\mathrm{A}}=6.53, \delta_{\mathrm{B}}=7.02,{ }^{3} \mathrm{~J}_{\mathrm{AB}}=8.2 \mathrm{~Hz}, 4 \mathrm{H}\right.$, phenyl ring $\left.\mathrm{H}\right), 7.20-7.58 \mathrm{ppm}(\mathrm{m}, 5 \mathrm{H}$, aromatic $\mathrm{H})$; EI-MS m/z (\%): $352\left(\mathrm{M}^{+}, 2\right), 254\left(\mathrm{M}^{+}-\mathrm{C}_{6} \mathrm{H}_{10} \mathrm{O}, 94\right), 216\left(\mathrm{M}^{+}-\mathrm{CH}_{3} \mathrm{OC}_{6} \mathrm{H}_{4} \mathrm{CO}, 100\right), 149\left(\mathrm{M}^{+}-\right.$ $\left.\mathrm{C}_{6} \mathrm{H}_{5} \mathrm{CO},-\mathrm{C}_{6} \mathrm{H}_{10} \mathrm{O}, 90\right), 135\left(\mathrm{CH}_{3} \mathrm{OC}_{6} \mathrm{H}_{4} \mathrm{CO}^{+}, 52\right), 119\left(\mathrm{M}^{+}-\mathrm{CH}_{3} \mathrm{OC}_{6} \mathrm{H}_{4} \mathrm{CO}, 93\right), 105\left(\mathrm{C}_{6} \mathrm{H}_{5} \mathrm{CO}^{+}, 97\right)$, $77\left(\mathrm{C}_{6} \mathrm{H}_{5}{ }^{+}, 88\right)$; Anal. Calcd. for $\mathrm{C}_{22} \mathrm{H}_{24} \mathrm{O}_{4}$ (352.43): C, 74.97; H, 6.86. Found: C, 74.69; H, 6.89.

Reaction of 1-p-methoxyphenyl-3-phenyl-2,3-epoxy-1-propanone (1e). PTLC (10:1 petroleum etherethyl acetate, four times): Zone 1, recovered 1e (20 mg, 10\%); Zone 2, $86 \mathrm{mg} \mathrm{(30 \% )} \mathrm{of} \mathrm{cis-3-(p-}$ methoxy-benzoyl)-4-phenyl-2,5-dioxaspiro[4,5]decane (7e), recrystallized from cyclohexane, m.p. 
129-130 ${ }^{\circ} \mathrm{C}$; IR cm ${ }^{-1}: 3045,2800-2980,1680,1600,1250,1120,1020 ;{ }^{1} \mathrm{H}-\mathrm{NMR}(\delta): 1.10-2.21$ (m, $10 \mathrm{H}$, cyclohexane $\mathrm{H}), 3.74\left(\mathrm{~s}, 3 \mathrm{H}, \mathrm{OCH}_{3}\right), \mathrm{AB}$-system $\left(\delta_{\mathrm{A}}=5.50, \delta_{\mathrm{B}}=5.74,{ }^{3} \mathrm{~J}_{\mathrm{AB}}=7.4 \mathrm{~Hz}, 2 \mathrm{H}, 4-\mathrm{H}, 3-\right.$ $\mathrm{H})$, AB-system $\left(\delta_{\mathrm{A}}=6.65, \delta_{\mathrm{B}}=7.44,{ }^{3} \mathrm{~J}_{\mathrm{AB}}=8.0 \mathrm{~Hz}, 4 \mathrm{H}\right.$, phenyl ring $\left.\mathrm{H}\right), 7.10 \mathrm{ppm}(\mathrm{s}, 5 \mathrm{H}$, aromatic $\mathrm{H})$; EI-MS $m / z(\%): 352\left(\mathrm{M}^{+}, 3\right), 254\left(\mathrm{M}^{+}-\mathrm{C}_{6} \mathrm{H}_{10} \mathrm{O}, 17\right), 217\left(\mathrm{M}^{+}-\mathrm{CH}_{3} \mathrm{C}_{6} \mathrm{H}_{4} \mathrm{CO}, 12\right), 149\left(\mathrm{M}^{+}-\mathrm{C}_{6} \mathrm{H}_{10} \mathrm{O}\right.$, $\left.-\mathrm{C}_{6} \mathrm{H}_{5} \mathrm{CO}, 4\right), 135\left(\mathrm{CH}_{3} \mathrm{OC}_{6} \mathrm{H}_{4} \mathrm{CO}^{+}, 100\right), 119\left(\mathrm{M}^{+}-\mathrm{C}_{6} \mathrm{H}_{10} \mathrm{O},-\mathrm{CH}_{3} \mathrm{OC}_{6} \mathrm{H}_{4} \mathrm{CO}, 36\right), 107\left(\mathrm{CH}_{3} \mathrm{OC}_{6} \mathrm{H}_{4}^{+}\right.$, 6), $105\left(\mathrm{C}_{6} \mathrm{H}_{5} \mathrm{CO}^{+}, 3\right), 77\left(\mathrm{C}_{6} \mathrm{H}_{5}^{+}, 16\right)$; Anal. Calcd. for $\mathrm{C}_{22} \mathrm{H}_{24} \mathrm{O}_{4}$ (352.43): C, 74.97; H, 6.86. Found: C, 75.07; H, 6.79.

Reaction of 1-p-bromophenyl-3-phenyl-2,3-epoxy-1-propanone (1g). PTLC (12:1 petroleum etherethyl acetate, three times): Zone 1, recovered $1 \mathrm{~g}$ (72 mg, 30\%); Zone 2, $76 \mathrm{mg}$ (24\%) of cis-3-(pbromobenzoyl)-4-phenyl-2,5- dioxaspiro[4,5]decane (7g), recrystallized from cyclohexane, m.p. 122$124{ }^{\circ} \mathrm{C}$; IR cm ${ }^{-1}: 3040,2880-2985,1690,1580,1240,1170,1110,1050 ;{ }^{1} \mathrm{H}-\mathrm{NMR}(\delta): 1.12-2.20(\mathrm{~m}$, $10 \mathrm{H}$, cyclohexane $\mathrm{H}), \mathrm{AB}$-system $\left(\delta_{\mathrm{A}}=5.60, \delta_{\mathrm{B}}=5.70,{ }^{3} \mathrm{~J}_{\mathrm{AB}}=7.4 \mathrm{~Hz}, 2 \mathrm{H}, 4-\mathrm{H}, 3-\mathrm{H}\right), 7.05(\mathrm{~s}, 5 \mathrm{H}$, phenyl ring $\mathrm{H}), 7.30 \mathrm{ppm}(\mathrm{s}, 4 \mathrm{H}$, aromatic $\mathrm{H})$; EI-MS $\mathrm{m} / z(\%): 402\left[\mathrm{M}^{+}\left({ }^{81} \mathrm{Br}\right), 6\right], 400\left[\mathrm{M}^{+}\left({ }^{79} \mathrm{Br}\right), 7\right]$, $304\left[\mathrm{M}^{+}\left({ }^{81} \mathrm{Br}\right)-\mathrm{C}_{6} \mathrm{H}_{10} \mathrm{O}, 40\right], 302\left[\mathrm{M}^{+}\left({ }^{79} \mathrm{Br}\right)-\mathrm{C}_{6} \mathrm{H}_{10} \mathrm{O}, 40\right], 296\left[\mathrm{M}^{+}\left({ }^{81} \mathrm{Br}\right)-\mathrm{C}_{6} \mathrm{H}_{5} \mathrm{CHO}, 33\right], 294\left[\mathrm{M}^{+}\right.$ ( $\left.\left.{ }^{79} \mathrm{Br}\right)-\mathrm{C}_{6} \mathrm{H}_{5} \mathrm{CHO}, 34\right], 217\left(\mathrm{M}^{+}-\mathrm{BrC}_{6} \mathrm{H}_{4} \mathrm{CO}, 73\right), 185\left({ }^{81} \mathrm{BrC}_{6} \mathrm{H}_{4} \mathrm{CO}^{+}, 98\right), 183\left({ }^{79} \mathrm{BrC}_{6} \mathrm{H}_{4} \mathrm{CO}^{+}, 100\right)$, $157\left({ }^{81} \mathrm{BrC}_{6} \mathrm{H}_{4}^{+}, 54\right), 155\left({ }^{79} \mathrm{BrC}_{6} \mathrm{H}_{4}^{+}, 54\right), 119\left(\mathrm{M}^{+}-\mathrm{C}_{6} \mathrm{H}_{10} \mathrm{O},-\mathrm{BrC}_{6} \mathrm{H}_{4} \mathrm{CO}, 97\right), 77\left(\mathrm{C}_{6} \mathrm{H}_{5}^{+}, 55\right)$; Anal. Calcd. for $\mathrm{C}_{21} \mathrm{H}_{21} \mathrm{BrO}_{3}$ (401.29): C, 62.85; H, 5.27. Found: C, 62.91; H, 5.24.

\section{References}

1. Schaap, A. P.; Siddiqui, S.; Prasad, G.; Maqsudur Rahman, A. F. M.; Oliver, J. P. Stereoselective Formation of Cis Ozonides by Electron-Transfer Photooxygenation of Naphthyl-Substituted Epoxides. Stereochemical Assignments of Ozonides by X-ray Crystallography and Chromatographic Resolution. J. Am. Chem. Soc. 1984, 106, 6087-6088.

2. Futamura, S.; Kusunose, S.; Ohta, H.; Kamiya, J. Photoinduced Electron Transfer Reaction. Part 3. 9,10-Dicyanoanthracene-sensitized Photo-oxidation of Electron-rich Stilbene Oxides. J. Chem. Soc. Perkin Trans 1, 1984, 15-19.

3. Miyashi, T.; Kamata, M.; Mukai, T. Simultaneous Capture of Two Distinct Radical Ion Intermediates Generated from the EDA Complexes of Three-Membered Compounds with TCNE by Photoexcitation and in the Dark. J. Am. Chem. Soc. 1987, 109, 2780-2788.

4. Masaki, Y.; Miura, T.; Ochiai, M. Alcoholysis of Epoxides Catalyzed by Tetracyanoethylene and Dicyanoketene Acetals. Bull. Chem. Soc. Jpn. 1996, 69, 195-205.

5. Hasegawa, E.; Ishiyama, K.; Kashiwazaki, H.; Horaguchi, T.; Shimizu, T. Selective $\mathrm{C}_{\beta}-\mathrm{O}$ Bond Cleavage of Chalcone Epoxides Induced by Pyrilium Salt Sensitized Photoreactions and Dark Reactions with Cerium(IV) Salts. Tetrahedron Lett. 1990, 31, 4045-4048.

6. Hasegawa, E.; Ishiyama, K.; Horaguchi, T.; Shimizu, T. Selective C $\alpha$-O Bond Cleavage of $\alpha, \beta-$ Epoxy Ketones to Aldols induced by Free Radical processes. J. Chem. Soc. Chem. Commun. 1990, 550-552. 
7. Hasegawa, E.; Ishiyama, K.; Kato, T.; Horaguchi, T.; Shimizu, T.; Tanaka, S.; Yamashita, Y. Photochemically and Thermally Induced Free-Radical Reactions of $\alpha, \beta$-Epoxy Ketones with Tributyltin Hydride: Selective C $\alpha-\mathrm{O}$ Bond Cleavage of Oxiranylmethyl Radicals Derived from $\alpha, \beta-$ Epoxy Ketones. J. Org. Chem. 1992, 57, 5352-5359.

8. Memarian, H. R.; Hesami, A.; Nikpour, F.; Döpp, D. Effect of Substituent on Photoinduced Ring Opening of $\alpha$-Epoxyketones by 2,4,6-Triphenylpyrylim Tetrafluoroborate (TPT). Indian J. Chem. Sec. B. 2001, 40B, 662-666.

9. Kagan, J.; Juang, P. Y.; Firth, B. E.; Przybytek, J. T; Singh, S. P. Catalysis of the Ionic-like Photoaddition of Methanol to Epoxides by Fe(III). Tetrahedron Lett. 1977, 4289-4290.

10. Iranpoor, N.; Mohammadpour-Baltork, I. Mild, Efficient and Selective Opening of Epoxides with Alcohols Catalyzed by Ceric (IV) Ammonium Nitrate. Synth. Commun. 1990, 20, 2789-2797.

11. Iranpoor, N.; Mohammadpour-Baltork, I.; Shiriny Zardaloo, F. Ceric Ammonium Nitrate, an Efficient Catalyst for Mild and Selective Opening of Epoxides in the Presence of Water, Thiols and Acetic acid. Tetrahedron 1991, 47, 9861-9866.

12. Iranpoor, N.; Mohammadpour-Baltork, I. 2,3-Dichloro-5,6-dicyano-p-benzoquinone, an Efficcient, Mild, Neutral and Highly Regioselective Catalyst for Alcoholysis of Epoxides. Tetrahedron Lett. 1990, 31, 735-738.

13. Iranpoor, N.; Salehi, P. Highly Efficient, Regio-and Stereoselective Alcoholysis of Epoxides Catalyzed with Iron(III) Chloride. Synthesis, 1994, 1152-1154.

14. Memarian, H. R.; Amini, M. K.; Nikjah, S. (unpublished results).

15. Memarian, H. R.; Nikpour, F. Photocatalytic Ring Opening of $\alpha$-Epoxyketones: 1,3-Dioxolane Formation. Chem. Mon. Accepted.

16. Pretsch, E.; Clerc, T.; Seibl, J.; Simon, W. in Tabellen zur Strukturaufklärung organischer Verbindungen mit spektroskopischen Methoden; Springer Verlag: Berlin, 1981; p. H70.

17. Hesse, M.; Meier, H.; Zeeh, B. in Spektroskopische Methoden in der Organischen Chemie; George Thieme Verlag: Stuttgart, 1984; pp 140-144.

18. William, D. H.; Fleming, I. in Spectroscopic Methods in Organic Chemistry; McGraw-Hill Book Company: London, 1989, p 135.

19. Murrov, S. L. in Handbook of Photochemistry; Marcel Dekker: New York, 1973; p 79.

Sample Availability: Samples are available from MDPI

(C) 2002 by MDPI (http://www.mdpi.org). Reproduction is permitted for noncommercial purposes. 